\title{
The Self-Protective Nature of Implicit Identity and Its Relationship to Weight Bias and Short-Term Weight Loss
}

\author{
Robert A. Carels Nova Hinman Afton Koball Marissa Wagner Oehlhof Amanda Gumble \\ Kathleen M. Young
}

Department of Psychology, Bowling Green State University, Bowling Green, OH, USA

\section{Keywords}

Implicit identity · Weight bias - Implicit attitudes . Weight loss

\section{Summary}

Background/Aims: Research suggests that making overly positive self-evaluations is the norm rather than the exception. However, unlike other stigmatized groups, overweight individuals do not exhibit a positive in-group social identity and instead exhibit significant explicit, implicit, and internalized weight bias. Therefore, it is not known whether overweight/obese individuals will evidence self-enhancement on general traits (good, attractive), or on traits inconsistent with fat stereotypes (disciplined, active, healthy eater), on an assessment of implicit attitudes. Similarly, it is not known whether these ratings will be associated with preexisting levels of weight bias, gender, or short-term weight loss. Methods: At baseline, 53 overweight/obese adults $\left(B M I>27 \mathrm{~kg} / \mathrm{m}^{2}\right.$, mean $\mathrm{BMI}=37.3 \mathrm{~kg} / \mathrm{m}^{2}, \mathrm{SD}=6.6 \mathrm{~kg} / \mathrm{m}^{2}, 89 \%$ Caucasian, and $77 \%$ female) participating in a weight loss intervention completed measures of explicit and internalized weight bias as well as implicit weight bias and identity (self-other comparisons). Results: Although participants evidenced significant anti-fat attitudes, they implicitly identified themselves as significantly thinner, better, more attractive, active, disciplined, and more likely to eat healthy than 'other' people. Compared to men, women were less likely to view themselves as thin and attractive relative to others. Greater implicit anti-fat bias and implicitly seeing the self as thin relative to others was associated with less short-term weight loss. Conclusion: Despite evidence for explicit, implicit, and internalized weight bias, participants generally evidenced a positive implicit self-identity, including areas consistent with negative fat stereotypes.

\section{Introduction}

Research indicates that, unlike other stigmatized groups, overweight and obese individuals do not exhibit a positive ingroup social identity [1]. For example, obese individuals often evidence explicit anti-fat attitudes and internalized weight bias [2-4]. Explicit attitudes, which are assessed through selfreport measures, typically reflect personal beliefs [5], while internalized attitudes reflect attitudes attributed to the self [6]. Similarly, there is considerable evidence for strong implicit anti-fat attitudes among the obese [2-4]. Implicit attitudes are unconscious, automatically activated evaluations acquired from repeated messages in the environment [7]. These findings occur against the backdrop of a society obsessed with thinness and one that exacts weight and appearance standards on women that are virtually unattainable [8].

Despite evidence for internalized weight bias and its accompanying negative self-attributions, a body of psychological research on illusion and well-being [9] suggests that people generally make overly positive self-evaluations, exaggerate their perceptions of control and mastery, and exhibit unrealistic optimism. Similarly, implicit attitude research generally reveals that self-enhancement, or a tendency to maintain and enhance an overall evaluation of the self, is common. In fact, research utilizing the Implicit Associations Test suggests that in a 'me-other' comparison, the 'other' comparison target is viewed as more similar to a tyrant than as an 'average' person

\section{KARGER \\ Fax +497614520714 \\ Information@Karger.de}

www.karger.com (c) 2011 S. Karger GmbH, Freiburg

$1662-4025 / 11 / 0044-0278 \$ 38.00 / 0$

Accessible online at:

www.karger.com/ofa
Dr. Robert A. Carels

Department of Psychology, Bowling Green State University

Bowling Green, OH 43403, USA

Tel. +1 419 372-9405; Fax -6013

rcarels@bgsu.edu 
(e.g., much more negative than 'Santa Claus' and similar in valence to 'Hitler') [10]. These findings suggest that people automatically protect their sense of self-worth by choosing to compare him/herself to an 'other' that they are far superior to on a particular attribute.

Among overweight/obese individuals, it is unclear whether implicit and explicit anti-fat attitudes and internalized weight bias are associated with diminished implicit self-worth, such as general negative appraisals of one's goodness or attractiveness. Diminished implicit self-worth may also come in the form of specific appraisals consistent with popular stereotypes of obese individuals, such as the beliefs that obese individuals lack selfdiscipline, are sedentary, and eat unhealthy foods [2]. Because the self-enhancing nature of implicit attitudes occurs against the backdrop of widespread fat condemnation and its accompanying attributions of laziness and gluttony, it is not known ultimately which attitudes will prevail. Given the self-protective nature of implicit attitudes toward bolstering the self, it is even possible that overweight/obese people will not implicitly view themselves as fat. If their fat 'other' is severely obese, they may view themselves as thin, relatively speaking. In fact, there is some evidence that, at times, overweight people, particularly men, may implicitly view themselves as normal weight despite evidence to the contrary, possibly as a result of self-enhancement [11]. Under these conditions, it is not known whether overweight/obese individuals will view themselves as 'good', 'attractive', 'disciplined', 'active', or 'healthy eaters' relative to 'other' people, on an implicit attitude assessment.

The current study was exploratory in nature and collected from a convenience sample of overweight/obese treatmentseeking adults prior to beginning a weight loss program. It was designed to examine the relationships among explicit, internalized, and implicit anti-fat attitudes as well as implicit identity in the areas of 'thinness', 'attractiveness', 'goodness', 'discipline', 'activity', and 'healthy eating' as well as short-term weight loss in overweight/obese treatment-seeking men and women. As previous research suggests, we anticipated that overweight/obese weight loss participants would express some degree of explicit anti-fat attitudes as well as strong implicit anti-fat attitudes [2-4, 12]. Evidence of internalized weight bias was also anticipated, given that participants are surrounded by a culture that exalts thinness, despises fatness, considers body weight malleable, and regards excess weight as a moral failing [1]. Similarly, participation in a weight loss program is likely to indicate some dissatisfaction with excess fat, and prior research suggests that internalized weight bias may be common among the obese [6]. It was unclear whether the strong anti-fat attitudes and weight bias evident in overweight and obese individuals would be associated with a decreased sense of self-worth (as measured by tests of 'implicit identity') or if overweight and obese participants would exhibit selfenhancement in the face of negative attitudes toward the obese. Finally, given the disproportionate emphasis of thinness for women, gender differences were also explored.

\section{Participants and Methods}

\section{Participants}

53 overweight and obese adults participated in an 18-week weight loss intervention that examined the benefits of matching treatment intensity to participant performance (i.e., stepped care). Participants were recruited through local newspapers, e-mail, and a listserv at a Midwestern university. Participants were included if they were i) overweight/obese $\left(\mathrm{BMI} \geq 27 \mathrm{~kg} / \mathrm{m}^{2}\right.$ ) and ii) nonsmokers. Participants were excluded if they reported i) past or current cardiovascular disease, ii) insulin-dependent diabetes, or iii) musculoskeletal problems that would prevent participation in moderate physical activity. Participants received no incentives for participating and provided a USD 100.- deposit to reduce attrition, which was refunded following completion of the program. This investigation received full human subjects review board approval, and all participants provided documentation of their physician's medical clearance prior to starting the intervention.

Most participants were Caucasian $(88.5 \% ; \mathrm{N}=46)$ and female $(77.4 \% ; \mathrm{N}=41)$, and the mean age of participants was $47.15 \pm 14.1$ years $($ mean \pm SD). Annual income exceeded USD 30,000.- for approximately $70 \%(\mathrm{~N}=35$, not reported $=2)$ of the participants, and $73 \%(\mathrm{~N}=38$, not reported $=1$ ) had at least a baccalaureate degree. BMI at baseline was $37.3 \pm 6.6 \mathrm{~kg} / \mathrm{m}^{2}$ (range $27.4-61.3 \mathrm{~kg} / \mathrm{m}^{2} .37$ participants $(70 \%)$ completed the weight loss intervention.

\section{Study Design}

Participants began the 18-week program in a self-help portion of the program and were transitioned to more intensive treatments (i.e., weekly weight loss groups, individual counseling) at the 6th or 12th week if they did not meet predetermined weight loss goals. At an orientation session, participants were given measures of explicit, internalized, and implicit anti-fat attitudes as well as a measure of implicit identity. Short-term weight loss (following 6 weeks of baseline self-help) was also measured. Participants completed these same measures a second time at the end of the program; however, pre-post changes in these measures are not reviewed in this article. All assessments and interventions were conducted by a licensed clinical health psychologist or psychology doctoral students with experience leading weight loss interventions. The self-help portion of the program was based on the LEARN program [13] which is a comprehensive, empirically supported approach to weight management $[14,15]$. The program encourages gradually losing weight, progressively increasing physical activity, and decreasing energy and fat intake through permanent lifestyle changes.

\section{Measures}

Body weight was measured using a digital scale (BF-350e; Tanita, Arlington Heights, IL, USA) to the nearest $0.1 \mathrm{lb}$ and height was measured to the nearest 0.5 inch using a height rod on a standard spring scale.

\section{Implicit Bias and Implicit Identity}

The Implicit Attitudes Test (IAT) is a widely used tool to assess implicit attitudes by using reaction time to measure underlying automatic evaluations [16]. The primary principle behind the IAT is that biased attitudes consist of concepts that are linked more strongly to each other in a person's mind, and the strength of their linkage is shown when people typically place items into the same conceptual category more quickly when they are consistent with a biased attitude. Overall, research has indicated that the IAT is a valid indicator of implicit attitudes [17]. An electronic version of the IAT was used for this study [17]. Target category labels (i.e., fat people, thin people) and attribute category labels (i.e., good, bad; motivated, lazy) were paired on the top of the computer screen (e.g., fat people/bad, thin people/good). Participants then classified a list of words (subordinate stimuli, e.g., fat, slim, terrible, wonderful) into their appropriate category as quickly and accurately as possible. To study implicit 
identity, the target categories 'self' and 'other' were paired with attribute category labels (thin, attractive, good, disciplined, active, and healthy eater). On some trials, the target and attribute categories were paired in a way to match expected implicit negative associations with weight (matched conditions), and on other trials, target and attribute pairings were reversed (mismatched conditions).

Individuals were told not to correct any errors. Prior to the weightbased and identity-based IATs, participants completed a practice IAT. Consistent with prior research, implicit bias was calculated using the algorithm outlined in Greenwald et al. [17].

\section{Internalized Bias}

The Weight Bias Internalization Scale (WBIS) [6] is an 11-item measure that examines level of internalization of anti-fat attitudes in overweigh and obese individuals. In traditional measures of anti-fat attitudes, it has been assumed that when an overweight or obese person completes these measures, their level of bias is equivalent with how they feel about themselves. Durso and Latner [6] argue, however, that using these traditional measures may only be examining overweight and obese persons' views about obesity in general, but not necessarily with regard to themselves. The WBIS utilizes a 7-point Likert scale ranging from 'strongly disagree' to 'strongly agree.' Participants are asked to rate their agreement with items such as, 'As an overweight person, I feel that I am just as competent as anyone.' Internal consistency for the WBIS in the initial creation of the scale was found to be good (Cronbach's $\alpha=0.90$ ) [6], and in this investigation Cronbach's $\alpha$ was 0.92 .

\section{Explicit Bias}

The Obese Person Trait Survey (OPTS) is a 20-item scale that measures stereotypical traits about obese persons [19]. Ten negative traits (lazy, undisciplined, gluttonous, self-indulgent, unclean, lack of willpower, unattractive, unhealthy, insecure, sluggish) and 10 positive traits (honest, generous, sociable, productive, organized, friendly, outgoing, intelligent, warm, humorous) are included. Participants were instructed to estimate the percentage (between 0 and 100) of obese people who possess each particular trait. In this investigation, participants were asked to complete the survey a second time and estimate the percentage of normal-weight persons who possess these traits as well as their confidence in their estimates. The OPTS has been found to be reliable [19]. For this study, Cronbach's $\alpha$ values were 0.88 for negative stereotypes of obese persons, 0.84 for positive stereotypes of obese persons, 0.90 for negative stereotypes of normal-weight persons, and 0.86 for positive stereotypes of normal-weight persons.

\section{Data Analyses}

T-tests and Pearson correlations were used to examine the association between demographic factors, including BMI, implicit identity, and weight bias. One-sample t-tests were used to examine levels of implicit weight bias and implicit attitudes. T-tests were used to examine differences among implicit attitudes as well as gender differences on explicit, implicit, and internalized weight bias. Pearson correlations were used to examine the association among implicit identity as well as between implicit identity and explicit, implicit, and internalized weight bias and between implicit identity, weight bias, and short-term weight loss.

\section{Results}

Very few associations were found between demographic variables (including BMI) and weight bias and implicit identity. Because there were only 6 non-Caucasian adults, statistical tests between Caucasian and non-Caucasian individuals were not run. As education increased, internalized weight bias decreased $(\mathrm{r}=-0.32, \mathrm{p}=0.03)$. As income increased, attributing positive traits to obese individuals decreased ( $\mathrm{r}=-0.44, \mathrm{p}<0.01)$. BMI was unrelated to internalized weight bias, explicit weight bias, implicit weight bias, and attitudes toward self.

\section{Implicit, Explicit, and Internalized Weight Bias}

On the IAT, response time was significantly shorter when the target category 'fat people' was paired with the attribute category 'bad' versus the attribute category 'good' $(\mathrm{M}=0.11$, SD $=0.09 ; \mathrm{t}(52)=8.7, \mathrm{p}<0.01)$ suggesting significant implicit anti-fat attitudes. For explicit weight bias, participants' estimates of obese people's positive attributes compared to estimates of normal-weight people's positive attributes were not significantly different. However, participants estimated obese people to have significantly more negative attributes than normal-weight individuals, $\mathrm{t}(52)=-5.92, \mathrm{p}<0.01$ (obese: $\mathrm{M}=$ 57.6, $\mathrm{SD}=15.6$; normal weight: $\mathrm{M}=39.9, \mathrm{SD}=14.5)$. The average score on the internalized weight bias scale was 4.42 (SD $=1.6$ ), indicating moderate levels of internalized weight bias. Men and women did not differ on implicit, explicit, or internalized weight bias scores.

\section{Implicit Identity Attitudes}

On the IAT, response time was significantly shorter when the target category 'thin' was paired with the attribute category 'self' versus the attribute category 'other' $(\mathrm{t}(52)=4.85$, $\mathrm{p}<0.01)$. Similarly, response time was significantly shorter when the target category 'good' was paired with the attribute category 'self' versus the attribute category 'other' $(\mathrm{t}(52)=$ $5.67, \mathrm{p}<0.01$ ) and when the target category 'attractive' was paired with the attribute category 'self' versus the attribute category 'other' $(\mathrm{t}(52)=8.46, \mathrm{p}<0.01)$. Finally, response time was significantly shorter when the target categories 'active', 'disciplined', and 'healthy eater' were paired with the attribute category 'self' versus the attribute category 'other' $(\mathrm{t}(52)=6.95, \mathrm{p}<0.01 ; \mathrm{t}(52)=8.06, \mathrm{p}<0.01$; and $\mathrm{t}(52)$ $=8.00, \mathrm{p}<0.01$, respectively). These findings suggest that participants implicitly identified themselves as significantly thinner, better, more attractive, active, disciplined, and more likely to eat healthy than other people (table 1).

Women were significantly less likely to view themselves as thin (men: $\mathrm{M}=0.08, \mathrm{SD}=0.07$; women: $\mathrm{M}=0.05, \mathrm{SD}=0.07$; $\mathrm{F}(2.53)=3.02, \mathrm{p}=0.044)$ and attractive (men: $\mathrm{M}=0.14, \mathrm{SD}=$ 0.05 ; women: $\mathrm{M}=0.09, \mathrm{SD}=0.09 ; \mathrm{F}(2.53)=3.10, \mathrm{p}=0.042$ ) than were men. Men and women did not differ on associations with the other categories.

Comparisons among implicit attitudes indicated that relative to others, participants more strongly identified themselves as attractive $(\mathrm{M}=0.10, \mathrm{SD}=0.09)$, active $(\mathrm{M}=0.9, \mathrm{SD}$ $=0.09)$ and a healthy eater $(\mathrm{M}=0.10, \mathrm{SD}=0.09)$, than they identified themselves as $\operatorname{good}(\mathrm{M}=0.05, \mathrm{SD}=0.07)$, thin $(\mathrm{M}=0.05, \mathrm{SD}=0.07)$, and disciplined $(\mathrm{M}=0.07, \mathrm{SD}=0.06)$. 
In other words, individuals revealed a stronger identification with the attributes of being attractive, active, and a healthy eater than with the attributes of being good, thin, and disciplined (table 1).

\section{Correlations among Implicit Attitudes, Internalized Weight Bias, and Explicit Attitudes}

Greater internalized weight bias was associated with viewing obese people as possessing negative traits $(\mathrm{r}=0.46, \mathrm{p}<0.01)$. Viewing obese people as possessing positive traits was not associated with internalized weight bias or viewing obese people as possessing negative traits. However, the more likely an individual viewed obese people as possessing negative traits on the OPTS, the more likely they were to view themselves as active $(\mathrm{r}=0.31, \mathrm{p}<0.02)$ and disciplined $(\mathrm{r}=0.25, \mathrm{p}<0.05)$ on the IAT.

On implicit attitudes, the more strongly individuals identified themselves as thin, the more strongly they identified themselves as a healthy eater $(\mathrm{r}=0.34, \mathrm{p}<0.01)$ and viewed fat as bad $(\mathrm{r}=0.27, \mathrm{p}<0.03)$. Also, the more likely an individual saw him/herself as attractive, the more likely he/ she was to view him/herself as good $(r=0.23, \mathrm{p}<0.05)$, selfdisciplined $(\mathrm{r}=0.39, \mathrm{p}<0.01)$, and a healthy eater $(\mathrm{r}=0.34$,

Table 1. Implicit bias and identity assessment

\begin{tabular}{lll}
\hline Categories & Mean & SD \\
\hline Thin-fat / good-bad $^{1}$ & 0.117 & 0.099 \\
Self-other / good-bad $^{2,3,4,5,6}$ & 0.052 & 0.067 \\
Self-other / healthy eater-unhealthy eater $^{2}$ & 0.100 & 0.091 \\
Self-other / active-inactive $^{2}$ & 0.084 & 0.088 \\
Self-other / thin-fat $^{2,3,4,5,6}$ & 0.050 & 0.075 \\
Self-other / attractive-unattractive $^{2}$ & 0.102 & 0.088 \\
Self-other / disciplined-undisciplined $^{2,3,4,5}$ & 0.066 & 0.060 \\
\hline
\end{tabular}

${ }^{1}$ Positive bias for thin; $\mathrm{p}<0.001$

${ }^{2}$ Positive bias for self; $\mathrm{p}<0.001$

Lower bias than Good-bad ${ }^{3}$, thin-fat ${ }^{4}$, disciplined-undisciplined ${ }^{5}$, and active-inactive ${ }^{6} ; \mathrm{p}<0.05$. $\mathrm{p}<0.01)$. Finally, seeing oneself as active was associated with seeing oneself as disciplined $(\mathrm{r}=0.47, \mathrm{p}<0.01)$ and seeing oneself as good was associated with seeing oneself as a healthy eater $(\mathrm{r}=0.25, \mathrm{p}<0.01)($ table 2$)$.

\section{Implicit, Explicit, and Internalized Attitudes and Short-Term Weight Loss}

In the stepped-care design of the current intervention, all participants began a self-help weight loss program and were stepped up to greater intensity treatment at various time points during the program if they failed to meet weight loss goals. Because participants received different intensity treatment following week 6 , only the associations between implicit and explicit attitudes and short-term weight loss (i.e., weight loss at week 6) were examined. Mean weight loss at week 6 was $8.1 \mathrm{lbs}(\mathrm{SD}=4.4 \mathrm{lbs})$ or $3.6 \%$ ( $\mathrm{SD}=1.8 \%)$ of body weight. Weight loss data was removed for one participant who was noncompliant with the treatment protocol, missed nearly all treatment sessions, and gained $11 \mathrm{lbs}$ by the end of treatment.

Greater implicit anti-fat bias was significantly associated with a lower percentage of weight loss $(r=-0.33, p=0.04)$ at week 6 and a less overall weight loss $(r=-0.34, p=0.04)$ at week 6 controlling for the influence of baseline BMI. Similarly, the more likely the individual was to implicitly identify him/herself with being thin, the smaller percentage weight loss at week $6(\mathrm{r}=-0.34, \mathrm{p}=0.04)$.

\section{Discussion}

The significant physical and psychological costs of weight bias, both intra- and inter-personal, are becoming increasingly well-established [2-4]. Consistent with previous research [12, 20], overweight/obese treatment-seeking adults in this investigation evidenced significant explicit anti-fat attitudes, ascribing more negative attributes to obese people than to to normal-weight people. Similarly, participants endorsed a moderate level of internalized weight bias, indicating being at least somewhat influenced by the pervasive weight-based negative

Table 2. Correlations among implicit constructs

\begin{tabular}{|c|c|c|c|c|c|c|}
\hline & $\begin{array}{l}\text { Thin-fat / } \\
\text { good-bad }\end{array}$ & $\begin{array}{l}\text { Self-other / } \\
\text { good-bad }\end{array}$ & $\begin{array}{l}\text { Self-other / } \\
\text { thin-fat }\end{array}$ & $\begin{array}{l}\text { Self-other / } \\
\text { healthy eater- } \\
\text { unhealthy eater }\end{array}$ & $\begin{array}{l}\text { Self-other / } \\
\text { active-inactive }\end{array}$ & $\begin{array}{l}\text { Self-other / } \\
\text { attractive- } \\
\text { unattractive }\end{array}$ \\
\hline Self-other / good-bad & 0.09 & & & & & \\
\hline Self-other / thin-fat & $0.27 *$ & -0.02 & & & & \\
\hline Self-other / healthy eater-unhealthy eater & 0.02 & $0.25^{*}$ & $0.34 * *$ & & & \\
\hline Self-other / active-inactive & 0.06 & 0.04 & 0.23 & 0.05 & & \\
\hline Self-other / attractive-unattractive & 0.14 & $0.23 *$ & 0.20 & $0.34 * *$ & 0.22 & \\
\hline Self-other / disciplined-undisciplined & 0.03 & 0.17 & 0.14 & 0.08 & $0.47 * *$ & $0.39 * *$ \\
\hline
\end{tabular}

$* \mathrm{p}<0.05, * * \mathrm{p}<0.01$. 
attitudes and discrimination prevalent in Western society. Not surprisingly, greater internalized weight bias was significantly associated with the view that obese people possess negative traits. Additionally, as predicted, significant implicit anti-fat attitudes were also observed among participants. Participants in this study did not differ by gender on any measures of weight bias. Past studies examining gender differences in weight bias have been equivocal [1, 21].

The significant anti-fat attitudes prevalent in this study's sample provide support for the assertion that overweight/ obese individuals do not demonstrate a positive in-group identity in contrast to other stigmatized groups [1]. However, consistent with research concerning self-enhancement in the general population [9], evidence for a self-enhancing effect was observed in this sample's self-appraisals of goodness and attractiveness as well as self-appraisals specifically related to common stereotypes about obese people. In implicit appraisals of self-worth, individuals more strongly viewed themselves as thin, good, attractive, active, disciplined, and healthy eaters compared to imagined 'other' people.

Several associations between implicit self-appraisals were significant, suggesting a correspondence between aspects of self-identity. For example, not surprisingly, the more likely an individual saw him/herself as thin, the more likely he/she was to view him/herself as a healthy eater, and the more likely an individual saw him/herself as active, the more likely he/she was to view him/herself as disciplined. In addition, consistent with findings from prior research on person perception and positive physical attractiveness stereotypes [22], viewing oneself as attractive in this investigation was associated with viewing the self as good, disciplined, and a healthy eater. While there is likely to be a different mechanism by which otherattractiveness stereotypes and self-attractiveness attitudes are formed, there was nevertheless consistency in the findings. Finally, seeing the self as 'good' was associated with seeing the self as a 'healthy eater'. Viewing oneself as a 'good' person because he/she eats healthy may reflect subtle self-condemnation for consuming unhealthy foods, or perhaps, reflect the negative portrayal of unhealthy foods in the media. These findings need to be replicated in future research.

In comparison to men, women were less likely to view themselves as thin or as attractive compared to other people. This result is not surprising, as previous research has shown that women face increased pressure to achieve an ideal of thinness and attractiveness that has been deemed unrealistic [8]. This finding is consistent with prior research showing that overweight men, compared to women, are more likely to implicitly view themselves as thin [11]. Although significant evidence for self-enhancement was found in this study, having a positive self-identity was not associated with lower implicit weight bias. In fact, it seems as though the opposite is true; implicitly viewing fat as bad was associated with implicitly appraising the self as thin. Additionally, viewing obese people as possessing negative traits was associated with implicitly viewing the self as active and disciplined. It appears that individuals with the highest levels of anti-fat attitudes and implicit weight bias are those that are most likely to protectively engage in self-enhancement strategies.

The pervasive effect of anti-fat bias is evident in weight loss outcomes as well, such that anti-fat bias may serve as a barrier to successful weight loss. Consistent with past research [20], the greater the implicit anti-fat bias, the lower the percentage weight loss and overall weight loss. Because self-appraisal of thinness and implicit anti-fat attitudes were related, it is also not surprising that the more likely an individual appraised oneself as being thin, the smaller the percentage of weight loss.

This investigation has several limitations that should be noted. The modest sized, self-selected sample had limited power to detect relationships among constructs and may limit the generalizibility of results to other overweight/obese individuals attempting to lose weight other ways (i.e., on their own or through another professional program) or those not attempting to lose weight. Additionally, the predominantly Caucasian and middle class sample suggests that replication with a larger, more diverse sample is necessary. Although relationships between explicit and implicit weight bias, internalization of weight bias, implicit identity, and weight loss outcomes were found, it is impossible to determine a causal relationship between the variables. Although the stepped-care design of the current investigation precluded an examination of weight bias and long-term weight loss, it would be of interest to measure the relationships among weight bias and selfenhancement with long-term weight loss.

In this investigation, participants did not complete an IAT that paired the target categories of 'fat/thin' with the attribute categories investigated in the identity IATs (e.g. 'healthy eater / unhealthy eater', 'active/inactive', etc.); so a direct comparison between these implicit anti-fat attitudes and the identities cannot be conducted. Showing that individuals more readily associate 'unhealthy eater, inactive, unattractive' with 'fat' (not done in this investigation), while pairing the opposite categories of 'healthy eater, active, and attractive' with the self (actual findings from this investigation) could provide more support for the self-enhancement theory. Another limitation of this study is that it is unclear who participants imagined as the 'other' in the identity IATs. Although previous research [10] has shown that individuals imagine an 'other' that is less desirable than them in specific attribute categories (e.g., more overweight, less attractive), the actual 'other' is unverified in the current investigation. Finally, although widely accepted procedures for creating, scoring, and interpreting the IAT [17] were used, the identity IAT used in this study is novel and has not been commonly used.

Moreover, most notably, in the face of significant explicit and implicit anti-fat attitudes and internalized weight bias, overweight/obese individuals may automatically engage in self-protection strategies to preserve a sense of self-worth. It is encouraging that although overweight and obese individu- 
als do not exhibit a positive in-group social identity, individually they maintain a positive implicit identity through self-enhancement strategies. The current investigation provides an initial response to a question posed by previous research [20] in which a connection was found between weight bias and adverse psychological and physical effects, namely: 'What are the underlying mechanisms for these effects?' Previous research has postulated that overweight/obese individuals perceive the negative traits identified in measures of bias to be true of themselves [12]. The current investigation suggests that there may be other reasons underlying the connection between weight bias and adverse outcomes. Subsequent research in bias-reducing interventions might target pervasive anti-fat attitudes while being sensitive to self-enhancement strategies that may be buffering the adverse effects of bias.

\section{Disclosure Statement}

The authors declared no conflict of interest.

\section{References}

1 Puhl RM, Heuer CA: The stigma of obesity: a review and update. Obesity 2009;17:1-24.

$\checkmark 2$ Puhl RM, Moss-Racusin CA, Schwartz MB: Internalization of weight bias: implications for binge eating and emotional well-being. Obesity 2007;15: $19-23$.

3 Wang SS, Brownell KD, Wadden TA: The influence of the stigma of obesity on overweight individuals. Int J Obes 2004;28:1333-1337.

4 Crandall CS: Prejudice against fat people: ideology and self-interest. J Pers Soc Psychol 1994;66:882-894.

$\checkmark 5$ Wilson TD, Lindsey S, Schooler TY: A model of dual attitudes. Psychol Rev 2000;107:101-126.

6 Durso LE, Latner JD: Understanding self-directed stigma: development of the Weight Bias Internalization Scale. Obesity 2008;16(suppl 2):S80-86.

7 Dovidio JF, Kawakami K, Gaertner SL: Implicit and explicit prejudice and interracial interaction. $\mathrm{J}$ Pers Soc Psychol 2002;82:62-68.

$\checkmark$ Thompson JK, Stice E: Thin-ideal internalization: mounting evidence for a new risk factor for bodyimage disturbance and eating pathology. Curr Dir Psychol Sc 2001;10:181-183.

9 Taylor SE, Brown JD: Illusion and well-being: a social psychological perspective on mental health. Psychol Bull 1988;103:193-210.
10 Karpinski A: Measuring self-esteem using the Implicit Associations Test: the role of the other. Pers Soc Psychol Bull 2004;30:22-34.

11 Grover VP, Keel PK, Mitchell JP: Gender differences in implicit weight identity. Int J Eat Disord 2003;34:125-135.

12 Carels RA, Young KM, Coit C, Harper J, Gumble A, Wagner Oehlhof, M, et al: Internalized weight stigma and its ideological correlates among treatment-seeking adults. Eat Weight Disord 2011:(in press).

13 Brownell KD: The LEARN Program for Weight Management, 10th ed. Dallas, American Health Publishing Company, 2004.

14 Andersen R, Wadden T, Bartlett S, Zemel B, Verde T, Franckowiak S: Effects of lifestyle activity vs. structured aerobic exercise in obese women. JAMA 1999;281:335-340.

15 Wadden T, Foster G, Letizia K: One-year behavioral treatment of obesity: comparison of moderate and sever caloric restriction and the effects of weight maintenance therapy. J Consult Clin Psychol 1994;62:165-171.
16 Greenwald AG, McGhee DE, Schwartz JLK Measuring individual differences in implicit cognition: The Implicit Association Test. J Pers Soc Psychol 1998;74:1464-1480.

17 Greenwald AG, Nosek BA, Banaji MR: Understanding and using the Implicit Association Test: I. An improved scoring algorithm. J Pers Soc Psychol 2003;85:197-216.

18 Teachman BA, Brownell KD: Implicit anti-fat bias among health professionals: is anyone immune? Int J Obes 2001;25:1525-1531.

19 Puhl RM, Schwartz M, Brownell KD: Impact of perceived consensus on stereotypes about obese people: a new approach for reducing bias. Health Psychol 2005;24:517-525.

20 Carels RA, Young KM, Wott CB, Harper J, Gumble A, Oehlhof MW, et al: Weight bias and weight loss treatment outcomes in treatment seeking adults. Ann Behav Med 2009;37:350-355.

21 Chen EY, Brown M: Obesity stigma in sexual relationships. Obes Res 2005;13:1393-1397.

22 Eagly AH, Ashmore RD, Makhijani MG, Longo LC: What is beautiful is good ...: a meta-analytic review of research on the physical attractiveness stereotype. Psychol Bull 1991;110:109-128. 